\title{
REA_07 - Selecting Aptamers for Hantaviruses Diagnostic
}

Valdez, E.C.N ${ }^{1 *}$.

${ }^{1}$ UERJ - Universidade Estadual do Rio de Janeiro.

Introduction: The occurrence of hantaviruses is increasing in Brazil, mainly causing a Cardiopulmonary Syndrome by Hantavirus (WHO, 2019). The disease treatment and collection of epidemiological data about virus dissemination require a diagnostic method. Results have shown significant Stern-Volmer constants differences which could elect a possible gold star one to the best performance in POC test.

Objective: This work proposed select aptamers against hantavirus Andes protein (ANDES) and then used fluorometric method to choose candidates for diagnostic agents among them.

\section{Methodology:}

\section{SELEX}

Aptamers selection applied a random DNA library to identify sequences that recognize the specific virus target, through the Systematic Evolution of Binding by Exponential Enrichment (SELEX) method according to Ellington, et al, 1990 and Tuerk, et al, 1990. Aptamers selection followed protocol of Simmons et al 2012.

\section{Fluorescence Quenching Experiments}

The decay of a fluorescence intensity gave us a method based on the suppression of a fluorophore energy when attached to a quencher agent, what was developed by Lackovicz and collaborators based on the Stern-Volmer Theory. The fluorescence quenching determination used $2 \mu \mathrm{M}$ solution of ANDES and HSA protein in phosphate buffer, titrated with increasing volumes of $1 \mu \mathrm{M}$ of two aptamers, named $\mathrm{C} 07$ and $\mathrm{C} 0203$. Final concentrations were $0,1,2,3,4,5,6,7,9$ and $11 \mu \mathrm{M}$. Experiments were performed at 25 and $37^{\circ} \mathrm{C}$ and the fluorescence spectra were recorded in the range of $300-400 \mathrm{~nm}$, excited at 290 $\mathrm{nm}$ wavelength. Both emission and excitation slits width were set at $5 \mathrm{~nm}$. Fluorescence measurements were recorded on Agilent Co Cary Eclipse Fluorimeter and optical spectroscopy were performed in a UV-visible Shimatzu-160A. Data were analyzed using Microcal Origin 6 software applying equations proposed by Cortez et al, 2002.

Results: Comparing the Stern-Volmer constants, a linear relationship at low concentrations between the ANDES and HSA proteins against aptamers has been observed. The Stern-Volmer constants demonstrated a significant difference between the molecular affinity of these proteins with aptamers $\mathrm{C} 07$ and $\mathrm{C} 0203$, highlighting the greater affinity of aptamers with the hantavirus protein.

Conclusion: Aptamers $\mathrm{C} 07$ and $\mathrm{C} 0203$ prove to be promising reaction agents in detection mechanisms.

Keywords: Hantavirus; Aptamers; Fluorometric 